\title{
Population Pharmacokinetics of Blinatumomab in Pediatric and Adult Patients with Hematological Malignancies
}

\author{
John David Clements ${ }^{1} \cdot$ Min Zhu $^{1,2} \cdot$ Mita Kuchimanchi $^{1,3} \cdot$ Bianca Terminello $^{1} \cdot$ Sameer Doshi ${ }^{1}$ (i)
}

Published online: 2 November 2019

(c) The Author(s) 2019

\begin{abstract}
Background and objectives Blinatumomab (BLINCYTO ${ }^{\circledR}$ ) is a novel bispecific T cell engager (BiTE ${ }^{\circledR}$ ) approved in the USA for the treatment of relapsed or refractory B cell precursor acute lymphoblastic leukemia (ALL) in children and adults, as well as minimal residual disease ALL in adults. This analysis characterized the population pharmacokinetics of intravenous blinatumomab in pediatric and adult patients.

Methods A total of 2417 serum concentrations of blinatumomab from 674 patients, including adult $(n=628)$ and pediatric patients $(n=46)$, from eight clinical studies were analyzed. The impact of covariates on pharmacokinetic parameters were explored, and significant covariates were further evaluated using a simulation approach.

Results Blinatumomab pharmacokinetics were described by a one-compartment linear model with first-order elimination, a clearance $(\mathrm{CL})$ of $2.22 \mathrm{~L} / \mathrm{h}$, and a central volume of $5.98 \mathrm{~L}$. A statistically significant effect of body surface area (BSA) on CL was observed. The smallest BSA of $0.37 \mathrm{~m}^{2}$ in the pediatric population was associated with a $63 \%$ reduction in blinatumomab systemic CL, relative to an adult patient with the median BSA $\left(1.88 \mathrm{~m}^{2}\right)$, supporting the use of BSA-based dosing in patients of lower bodyweight. The BSA effect was minimal, with a $\leq 25 \%$ change in CL over the range of BSA in adults, supporting no need for BSA-based dosing.

Conclusions Blinatumomab pharmacokinetics were adequately described by a one-compartment linear model with first-order elimination. No covariates other than BSA on CL were identified as significant. BSA-based dosing should be considered for lightweight patients to minimize inter-subject variability in blinatumomab exposure.
\end{abstract}

\section{Introduction}

B cell precursor acute lymphoblastic leukemia (ALL), minimal residual disease (MRD)-positive B cell precursor ALL, non-Hodgkin's lymphoma (NHL), Philadelphia chromosome-positive $(\mathrm{Ph}+)$ ALL, and childhood ALL represent the majority of difficult-to-treat lymphoid cancers. Despite different combinations of aggressive multidrug

Electronic supplementary material The online version of this article (https://doi.org/10.1007/s40262-019-00823-8) contains supplementary material, which is available to authorized users.

Sameer Doshi

sdoshi@amgen.com

1 Clinical Pharmacology, Modeling \& Simulation, Amgen Inc., Thousand Oaks, CA, USA

2 Present Address: Regeneron Pharmaceuticals, Tarrytown, NY, USA

3 Present Address: Biogen, Cambridge, MA, USA chemotherapies, many patients with these leukemias or lymphomas either fail to achieve complete remission or end up with relapsed or refractory $(\mathrm{R} / \mathrm{R})$ diseases, with only $20-40 \%$ of the adult population achieving long-term remission [1] and only $85-90 \%$ in the pediatric population being cured, albeit with relapse rates of $10-15 \%$ [2]. Thus, there remains a need for novel therapeutic approaches. For cancers of lymphoid origin, CD19 is an attractive target due to its high expression in most NHLs and many leukemias, including ALL, chronic lymphocytic leukemia, and hairy cell leukemia. As the CD19 marker is not lost during neoplastic transformation of a normal cell into a cancer cell, CD19 is an ideal immunological target for the treatment of clonal B cell diseases [3-6].

Blinatumomab (BLINCYTO ${ }^{\circledR}$, Amgen Inc., Thousand Oaks, CA, USA) is a novel single-chain antibody construct in the class of the bispecific $\mathrm{T}$ cell engager $\left(\mathrm{BiTE}^{\circledR}\right)$ against CD19/CD3 [7]. Blinatumomab mediates the formation of a synapse between the CD3+ T cell and the CD19+ target cell, transiently connecting malignant cells with $\mathrm{T}$ cells to 


\section{Key Points}

A population pharmacokinetic model suitably described blinatumomab concentrations after continuous intravenous infusions in children and adults with hematologic malignancies, including relapsed/refractory (Philadelphia chromosome-positive or -negative) acute lymphoblastic leukemia (ALL), minimal residual disease-positive B cell precursor ALL, and non-Hodgkin's lymphoma.

Dose adjustments based on body surface area (BSA) are not justifiable in pediatric and adult patients weighing at least $45 \mathrm{~kg}$.

BSA-based dosing is warranted in children and adults weighing less than $45 \mathrm{~kg}$.

induce T cell-mediated killing of the bound malignant cell. Consequently, granules containing granzymes and the poreforming protein perforin fuse with the $\mathrm{T}$ cell membrane and discharge their toxic content. Released perforin forms pores in the presence of extracellular calcium that are inserted into the cancer cell membrane. These pores can then serve as entry sites for granzymes and are responsible for release of cytosolic content, leading to lysis of the CD19+ target cells [7]. Blinatumomab is approved in the USA for the treatment of R/R B cell precursor ALL in adult and pediatric populations [8,9], as well as ALL with MRD [10]. It is currently under investigation for the treatment of NHL and its subtypes in adults [11].

Previously, the pharmacokinetics of blinatumomab were evaluated in several phase I and II clinical trials [11-16] in adults with NHL, MRD-positive ALL, R/R ALL, and R/R NHL. In these studies, blinatumomab exhibited linear and time-independent pharmacokinetics and fast clearance (CL) under continuous intravenous (cIV) infusion for 4-8 weeks per cycle over a dose range of either 5-90 $\mu \mathrm{g}$ / $\mathrm{m}^{2} /$ day or $9-112 \mu \mathrm{g} /$ day. Moreover, a population pharmacokinetic model in adult patients with Philadelphia chromosome-negative $(\mathrm{Ph}-) \mathrm{R} / \mathrm{R}$ ALL was previously reported $(n=365)$, in which blinatumomab concentration data were well-described by an open one-compartment linear pharmacokinetic model [17]. The typical value (geometric mean) of blinatumomab volume of distribution $\left(V_{\mathrm{d}}\right)$ in adult patients was estimated to be $3.40 \mathrm{~L}$. CL was multimodal, where $90 \%$ of patients had a typical CL value of $1.36 \mathrm{~L} / \mathrm{h}$, and $10 \%$ had a typical CL value of $5.49 \mathrm{~L} / \mathrm{h}$ with high inter-individual variability (IIV) (coefficient of variation 60\%) [17]. Pharmacokinetics were similar in patients with ALL and NHL and were not affected by patient demographics. Creatinine clearance (CrCL) was estimated using the Cockcroft-Gault formula [18] and was a significant covariate for drug CL; however, no dose adjustment was required in adult patients with mild or moderate renal impairment. The incidence of neutralizing anti-blinatumomab antibodies was reported as less than $1 \%$ [17].

In the present population pharmacokinetic analysis, the pharmacokinetics of blinatumomab were evaluated simultaneously in adult and pediatric patients with ALL for the first time $(n=674)$. Pharmacokinetic data from phase I, II, and III studies in patients with MRD-positive ALL and with $\mathrm{Ph}+$ and $\mathrm{Ph}-\mathrm{R} / \mathrm{R}$ ALL were included. The objectives of this analysis were to characterize the pharmacokinetics of blinatumomab in patients following cIV administration over the age range of 7 months to 80 years; quantify the degree of IIV in pharmacokinetic parameters and residual variability; evaluate patient-related covariates as potential sources of variability in the pharmacokinetics of blinatumomab and its relationship with selected dosing regimens; and conduct model-based simulations to support dose conversion between body surface area (BSA)-based dosing and fixed doses.

\section{Methods}

\subsection{Clinical Data}

Data from eight clinical studies (one phase I, one phase I/II, five phase II, and one phase III) in 674 patients were included in this analysis. Study populations included adult patients with relapsed NHL $(n=67)$, MRD-positive B-lineage ALL $(n=52), \mathrm{R} / \mathrm{R} \mathrm{Ph}-\mathrm{ALL}(n=472), \mathrm{R} / \mathrm{R} \mathrm{Ph}+\mathrm{ALL}(n=37)$, and pediatric patients with $\mathrm{R} / \mathrm{R} \mathrm{Ph}-\mathrm{ALL}(n=46)$. Data were pooled for this analysis (Table 1). Patients received blinatumomab as a cIV infusion over 4-8 weeks (multiple cycles) at dose ranges $0.5-90 \mu \mathrm{g} / \mathrm{m}^{2} /$ day and $9-28 \mu \mathrm{g} /$ day. Blinatumomab pharmacokinetics were assessed for up to ten cycles across studies. The relevant characteristics of each clinical study used for the current analyses are summarized in Table 1; additional details of these studies have been previously reported [11-15, 19-21].

\subsection{Bioanalysis}

Blinatumomab serum concentrations were assessed using a CD69 activation bioassay [22] with an upper limit of quantification of $1000 \mathrm{pg} / \mathrm{mL}$ and lower limit of quantification of 50-100 pg/mL (100 pg/mL in earlier studies: MT103-104 and MT103-202; and $50 \mathrm{pg} / \mathrm{mL}$ in later studies: MT103203, MT103-205, MT103-206, MT103-211, 20120216, and 00103311) [17]. The accuracy of the assay ranged from 73 to $123 \%$, and the precision of the assay, including at the lower limit of quantification, ranged from 72 to $120 \%$ within the linear range [17]. The basis of the assay is that during 


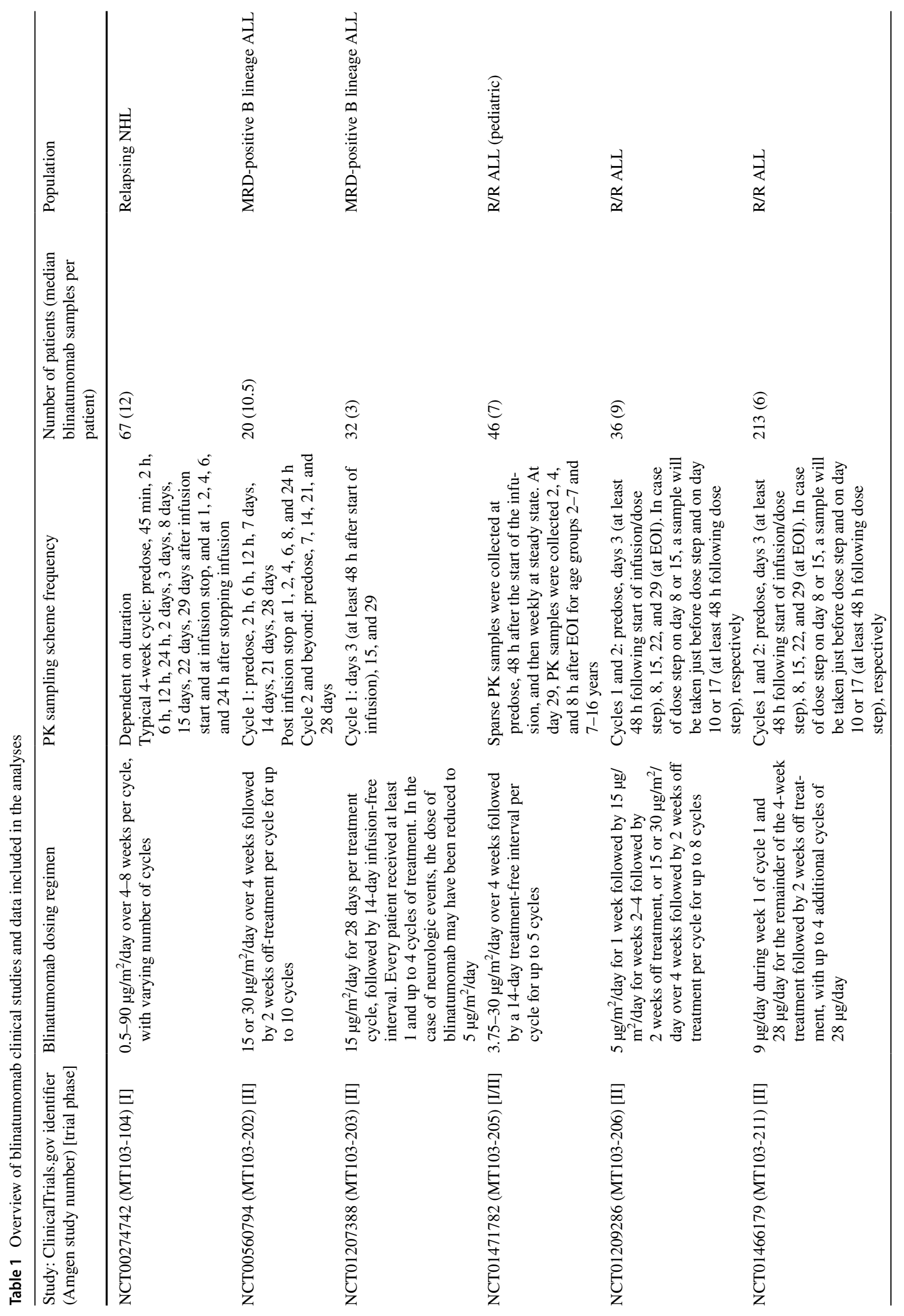




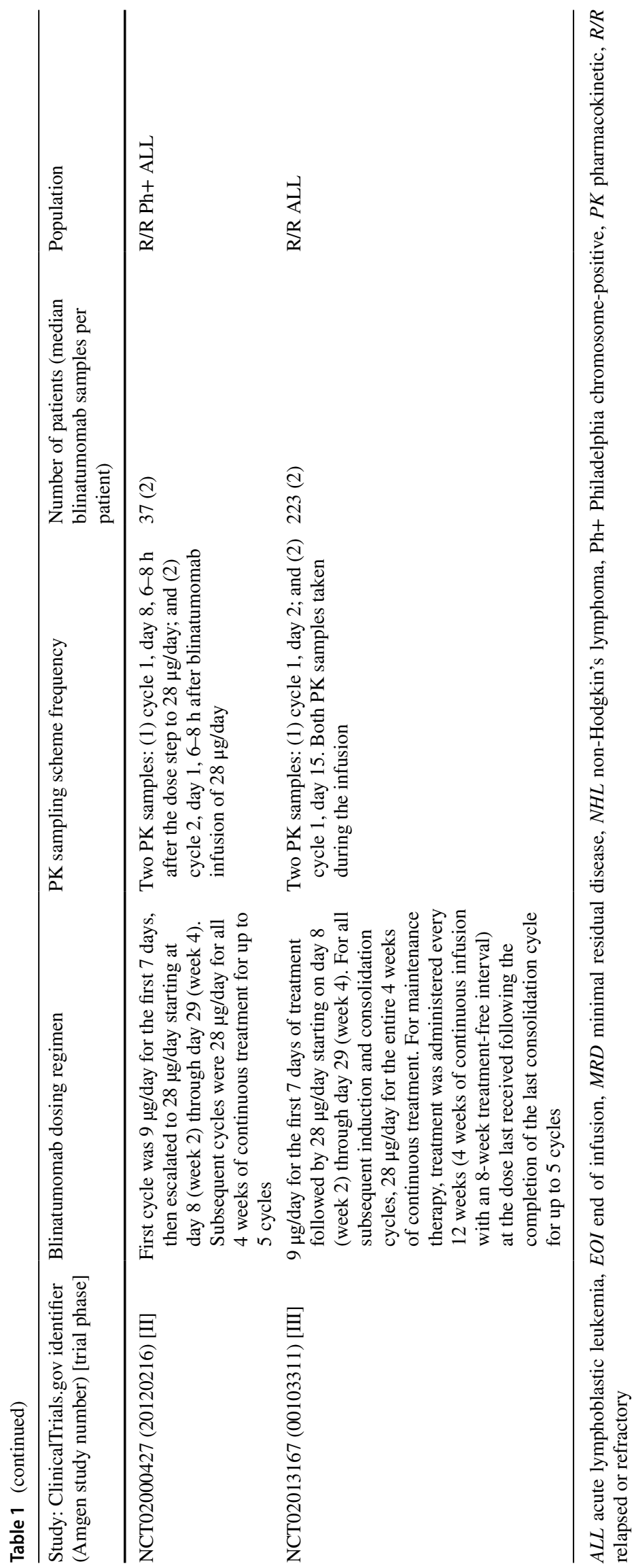


the blinatumomab-mediated cytotoxicity reaction, the CD69 activation marker is expressed on $\mathrm{T}$ cells in a blinatumomab concentration-dependent manner. In the assay, HPB-ALL cells ( $\mathrm{T}$ cell line) were incubated with Raji cells (B cell line) in the presence of serial blinatumomab dilutions in serum, ranging from $200 \mathrm{ng} / \mathrm{mL}$ to $3 \mathrm{pg} / \mathrm{mL}$, as previously published [17]. After incubation, T cells were labelled with mouse anti-CD69-FITC monoclonal antibody and measured in a fluorescence-activated cell sorting Canto unit. The forward scatter/side scatter mode was used for data analysis.

\subsection{Non-Compartmental and Exploratory Analyses}

Definitive non-compartmental analyses (NCAs) were conducted for clinical study report generation and are presented as stand-alone results but also used as an exploratory tool to better understand the data and any relationship between demographic and treatment-related factors with blinatumomab pharmacokinetic parameters. In this analysis, individual blinatumomab CL values, calculated as $R_{0} / C_{\mathrm{ss}}$, where $R_{0}$ is the infusion rate and $C_{\mathrm{ss}}$ is the average of $C_{\mathrm{ss}}$ (steadystate concentration) values available for an individual patient, were utilized to graphically evaluate any relationships between blinatumomab CL and BSA, age, race, and disease. Individual blinatumomab CL values, calculated as $R_{0} / C_{\mathrm{ss}}$ where $C_{\mathrm{ss}}$ is the individual $C_{\mathrm{ss}}$, were utilized to graphically evaluate any relationships between blinatumomab CL and dose and treatment cycle. One pediatric patient for the NCA analysis only, and not the population pharmacokinetic analysis, was excluded from the pharmacokinetic parameter reporting because the pharmacokinetic sample was from a retreatment cycle.

\subsection{Software for Population Pharmacokinetic Analysis}

Serum concentration versus time profiles were used for nonlinear mixed-effect modelling [23] using NONMEM ${ }^{\circledR}$ version 7.2 (ICON Development Solutions, Ellicott City, MD, USA) [24]. First-order conditional estimation (FOCE), FOCE interaction, and Laplacian methods were evaluated [25]. Graphical and all other statistical analyses, including evaluation of NONMEM ${ }^{\circledR}$ outputs, were performed using $\mathrm{R}$ version 3.0.1 or higher. In the exploratory analysis, blinatumomab pharmacokinetic parameters were also assessed using NCA with Phoenix WinNonlin ${ }^{\circledR}$ version 6.3 (Certara, Princeton, NJ, USA).

\subsection{Pharmacostatistical and Covariate Modeling}

Pharmacokinetic modeling was conducted on eight pediatric/adult clinical studies as outlined in Table 1. Full details regarding pharmacostatistical and covariate modeling methodologies are found in the Electronic Supplementary Materials.

\subsection{Internal Model Evaluation}

The final population pharmacokinetic model was internally evaluated using prediction-corrected visual predictive check (pcVPC) using 100 replicates [26] and non-parametric bootstrap methodologies using 500 replicates [27, 28]. Replication of the original dataset (a bootstrap sample) was obtained by random draws of individual data (with replacement) from the dataset. The population pharmacokinetic model was refitted to each new dataset. The stability of the model was evaluated by visual inspection of the distribution of the model parameter estimates obtained from the newly replicated datasets, and was compared with that obtained from the fit of the original dataset. The parameter estimates of the model were compared to the mean and $95 \%$ confidence interval (CI) of the model parameter obtained from the non-parametric bootstrap replicates that had been successfully minimized. If the parameter estimates were within the $95 \%$ CI obtained from the bootstrap analysis, the model was considered acceptable.

\subsection{Simulations}

As recommended in the prescribing information [8], blinatumomab is to be administered as a fixed dose for patients $\geq 45 \mathrm{~kg}$ and as a BSA-based dose for patients $<45 \mathrm{~kg}$. To compare fixed dosing ( $28 \mu \mathrm{g} /$ day $)$, BSAbased dosing $\left(15 \mu \mathrm{g} / \mathrm{m}^{2} /\right.$ day $)$, and per label-based $\left(15 \mu \mathrm{g} / \mathrm{m}^{2} /\right.$ day for patients $<45 \mathrm{~kg}$ and $28 \mu \mathrm{g} /$ day for patients $\geq 45 \mathrm{~kg}$ ) dosing strategies, 10,000 virtual patients were simulated from the final model using the multivariate distribution between bodyweight and BSA. Each simulated patient received each of the three dosing strategies. There were 1000 simulations for each $10 \mathrm{~kg}$ interval between 5 and $105 \mathrm{~kg}$ to ensure an equal number of patients across the bodyweight bins. The final model was then used to generate blinatumomab $C_{\mathrm{ss}}$, which was summarized in $10 \mathrm{~kg}$ bins.

\section{Results}

\subsection{Summary of Data for Population Pharmacokinetic Analysis}

A total of 674 patients in the combined population pharmacokinetic dataset received blinatumomab as a cIV infusion over 4-8 weeks for up to ten cycles at doses ranging from 0.5 to $90 \mu \mathrm{g} / \mathrm{m}^{2} /$ day and from 9 to $28 \mu \mathrm{g} /$ day. 
There were 4841 serum samples, $13(0.27 \%)$ had missing time, $287(5.93 \%)$ were collected more than 90 days after the start of infusion, and $248(5.12 \%)$ were collected more than 1 day after the end of the infusion, leaving 4293 samples. Of these 4293 samples, 664 (15.5\%) were below the lower limit of quantification, leaving 3629 serum samples in the analysis dataset. MRD patients constituted $7.7 \%$ of all patients available for analysis. The number of samples per patient, by study, is presented in Table 1. Individual concentration versus time profiles are shown in Electronic Supplementary Material Fig. 1.

One patient each had missing aspartate aminotransferase (AST) and alanine aminotransferase (ALT) values, two were missing total bilirubin, eight were missing serum albumin, and three were missing CrCL. Summary statistics for continuous and categorical covariates are shown in Electronic Supplementary Material Tables 1 and 2. Median age was 41.0 years (range 0.6-80). Median bodyweight was $70.7 \mathrm{~kg}$ (range 7.5-149), and median BSA was $1.8 \mathrm{~m}^{2}$ (range 0.4-2.70). In total, 406 men and 268 women were included in the analysis; $86 \%$ were white. Median CrCL was $121 \mathrm{~mL} / \mathrm{min}$ (range 36-150) calculated using the CockcroftGault equation for adult patients and the Schwartz equation for pediatric patients. Liver function was characterized by median values of $28.2 \mathrm{U} / \mathrm{L}$ for AST, $41.0 \mathrm{U} / \mathrm{L}$ for ALT, $37.1 \mathrm{~g} / \mathrm{L}$ for serum albumin, and $8.2 \mu \mathrm{mol} / \mathrm{L}$ of total bilirubin. Median lactate dehydrogenase (LDH) and hemoglobin were $297 \mathrm{IU} / \mathrm{L}$ and $10.1 \mathrm{~g} / \mathrm{dL}$, respectively.

\subsection{Non-Compartmental and Exploratory Analyses}

Graphical presentations for exploring relationships between blinatumomab CL and BSA, age, race, dose, treatment cycle, and disease are depicted in Fig. 1. Figures showing BSAnormalized CL by race, treatment cycle, dose, and disease are depicted in Electronic Supplementary Material Fig. 2. NCA results showed that blinatumomab CL (L/h) increased with BSA (Fig. 1a). Once the CL values were normalized by BSA in pediatric patients (Fig. 1b), they were similar across the age range, supporting BSA-based dosing in patients with lower BSA values. No apparent differences in the CL ranges across races were identified from this dataset (Fig. 1c). Blinatumomab CL values were consistent across treatment cycles (Fig. 1d) and dose levels (Fig. 1e). Blinatumomab was also similar across disease populations (Fig. 1f).

The summary of pharmacokinetic parameters generated by the NCA for patient groups weighing $\geq 45$ and $<45 \mathrm{~kg}$ (Table 2) indicates that with the recommended dosing regimens in the drug label, comparable $C_{\mathrm{ss}}$ values can be achieved for the two groups. A limited number of patients had the necessary intensive sampling to enable the estimation of volume of distribution based on terminal phase $\left(V_{\mathrm{z}}\right)$ and elimination half-life $\left(t_{1 / 2}\right)$, explaining the difference in the $n$ for the parameters. Based on these observations, the one-compartment model was consistent with the previously published model [17].

\subsection{Model Development}

The previously published model [17] reported a bimodal distribution in the predicted CL. When this model was applied to the new analysis, CL was no longer bimodal. Since the previous model was not externally valid for the new data, further model development was necessary to adequately characterize blinatumomab population pharmacokinetics.

Simplification of the model was achieved by removing the mixture model for two CL subpopulations, reducing to one residual error, and removing the IIV on the residual error. Therefore, the model used at the initiation of redevelopment had four parameters: $V_{\mathrm{d}}, \mathrm{CL}$, IIV on CL, and one residual error parameter. Based on assessment of empirical Bayes estimates (EBEs) versus BSA, BSA on CL was added to the model. This resulted in a significant decrease in the minimum value of the objective function of 35.6. Switching to an FOCE approach instead of a Laplacian approach (as previously utilized) further improved fitting. At this point in the model-building phase, the covariance step was not successful. When including IIV on the residual error, the model was stabilized, with a successful covariance step and a low condition number of 7. Inclusion of the effect of BSA on $V_{\mathrm{d}}$ resulted in unrealistic values of $V_{\mathrm{d}}\left(V_{\mathrm{d}}>9\right)$ and an unsuccessful covariance step. The model, now having $V_{\mathrm{d}}$, CL, BSA on CL, IIV on CL, residual variability, and IIV on residual variability, was used as the basis for which other covariates could be investigated.

The NCA and the visual and statistical analyses of the empirical Bayesian post hoc estimates for CL showed flat relationships, with no $r^{2}$ values higher than 3\% (Electronic Supplementary Material Figs. 3 and 4 with statistics in-figure). The lack of any meaningful relationships between CL random effects and covariates, combined with a low shrinkage value of $9 \%$, confirmed that it was unnecessary to test the effect of any covariate formally in NONMEM ${ }^{\circledR}$, including for dose level and treatment cycle.

With the addition of BSA as a covariate on CL, IIV on CL was decreased from 51.4 to $47.6 \%$. There were minor changes in CL (from 2.13 to $2.22 \mathrm{~L} / \mathrm{h}$ ), $V_{\mathrm{d}}$ (from 5.96 to $5.98 \mathrm{~L}$ ), IIV for residual error (from 63.9 to $64.3 \%$ ), and residual variability (from 55.5 to $55.9 \%$ ). There was no additional benefit to using a BSA covariate effect only in patients $<45 \mathrm{~kg}$ while keeping a fixed dosing approach in heavier patients, with the IIV on CL increasing from 47.6 to $47.8 \%$. In the final model, the shrinkage value for the random effects on residual variability was $5 \%$. The final model was a one-compartment linear pharmacokinetic 

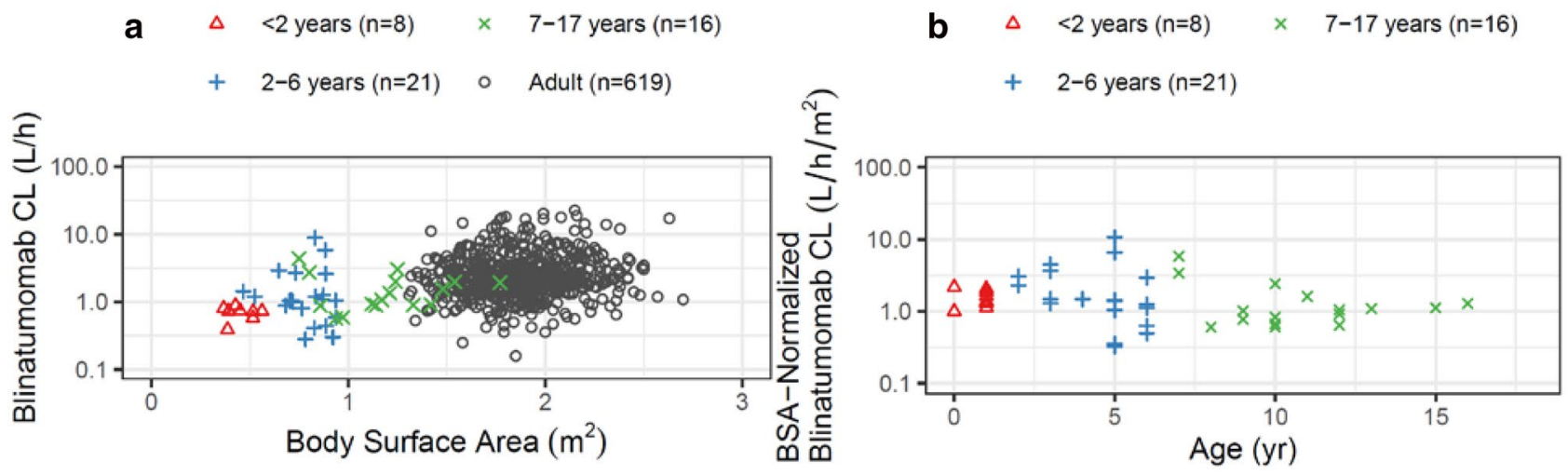

C

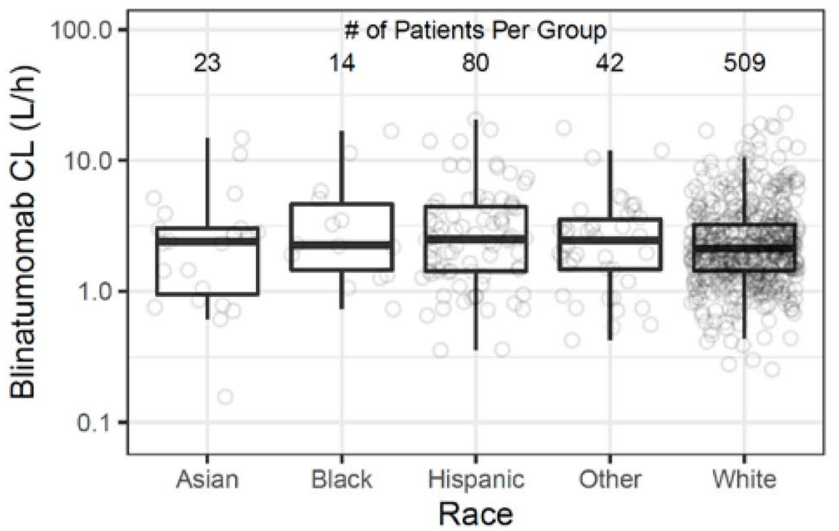

d

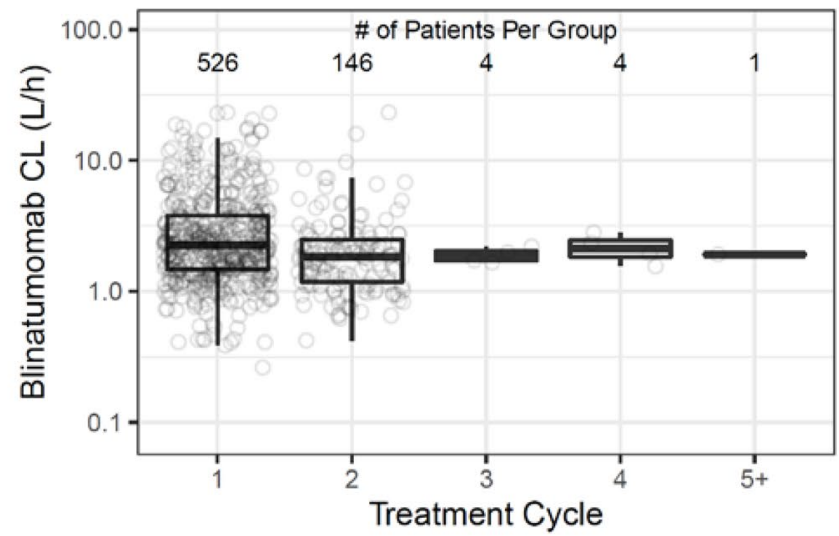

e
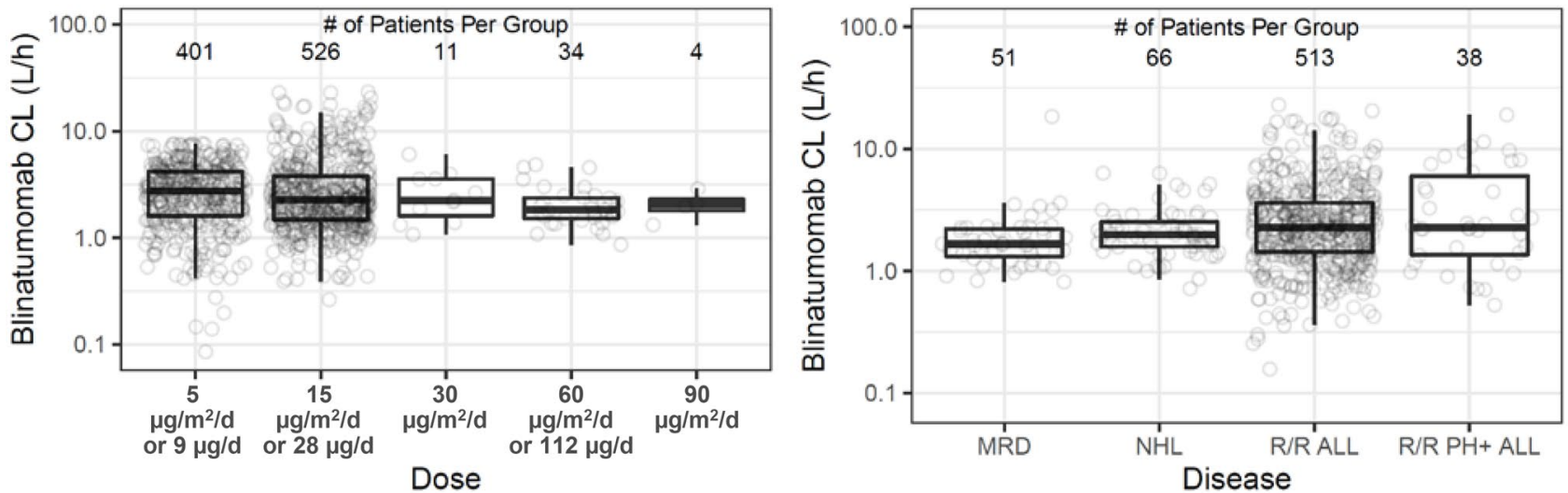

Fig. 1 Relationships between blinatumomab clearances and demographic, treatment, and disease-related factors. a Clearance versus body surface area. b Body surface area-normalized clearance versus age in pediatrics. c Clearance by race. $\mathbf{d}$ Clearance by treatment cycle after administration of $5 \mu \mathrm{g} / \mathrm{m}^{2} /$ day or $9 \mu \mathrm{g} /$ day in adults. e Clearance by dose after cycle 1 dose administration in adults. f Clearance by disease. The top, middle, and bottom of the boxes in figures $\mathbf{c}-\mathbf{f}$ are the third quartile, median, and first quartile, respectively. The whisk-

model parameterized in terms of systemic CL and $V_{\mathrm{d}}$. Pharmacokinetic parameters were assumed to be log-normally distributed, and an exponential IIV term was estimated for CL. Residual variability was modeled using an additive error model in the log-domain with IIV.

ers are drawn to the nearest value not beyond $1.5 \times$ (upper hingelower hinge). In figures $\mathbf{d}-\mathbf{e}$ individual blinatumomab clearance values were calculated as $\mathrm{R}_{0} / C_{\mathrm{ss}}$ where $C_{\mathrm{ss}}$ is the individual $C_{\mathrm{ss}}$. Height or weight data was not collected for four subjects in the clinical dataset. $A L L$ acute lymphoblastic leukemia, $B S A$ body surface area, $C L$ clearance, $C_{s s}$ steady-state concentration, $d$ day, $M R D$ minimal residual disease, $N H L$ non-Hodgkin's lymphoma, $P H$ Philadelphia chromosome, $R_{0}$ infusion rate, $R / R$ relapsed or refractory

Goodness-of-fit plots are shown in Electronic Supplementary Material Fig. 5. Observed versus predicted plots showed random normal scatter around the identity line, indicating the absence of systematic bias and the adequacy of the model to describe the data. In addition, conditional weighted 
Table 2 Blinatumomab noncompartmental pharmacokinetic parameter estimates following continuous intravenous infusion in patients with bodyweight $\geq 45$ and $<45 \mathrm{~kg}$

\begin{tabular}{|c|c|c|c|c|c|}
\hline Population & Clearance & $V_{\mathrm{z}}$ & $t_{1 / 2, \mathrm{Z}}$ & Dose & Cycle $1 C_{\mathrm{ss}}$ \\
\hline \multirow[t]{4}{*}{$\geq 45 \mathrm{~kg}$} & \multirow[t]{4}{*}{$\begin{array}{l}3.15(3.03) \mathrm{L} / \mathrm{h} \\
n=612\end{array}$} & \multirow[t]{4}{*}{$\begin{array}{l}4.40(2.53) \mathrm{L} \\
n=55\end{array}$} & \multirow[t]{4}{*}{$\begin{array}{l}2.10(1.41) \mathrm{h} \\
n=55\end{array}$} & $9 \mu \mathrm{g} /$ day & $\begin{array}{l}229(360) \mathrm{pg} / \mathrm{mL} \\
n=333\end{array}$ \\
\hline & & & & $28 \mu \mathrm{g} /$ day & $\begin{array}{l}615(538) \mathrm{pg} / \mathrm{mL} \\
n=395\end{array}$ \\
\hline & & & & $5 \mu \mathrm{g} / \mathrm{m}^{2} / \mathrm{day}$ & $\begin{array}{l}191(79.8) \mathrm{pg} / \mathrm{mL} \\
n=62\end{array}$ \\
\hline & & & & $15 \mu \mathrm{g} / \mathrm{m}^{2} /$ day & $\begin{array}{l}608(265) \mathrm{pg} / \mathrm{mL} \\
n=89\end{array}$ \\
\hline \multirow[t]{4}{*}{$<45 \mathrm{~kg}$} & \multirow[t]{4}{*}{$\begin{array}{l}1.87(1.96) \mathrm{L} / \mathrm{h} \\
n=56\end{array}$} & \multirow[t]{4}{*}{$\begin{array}{l}3.48(2.61) \mathrm{L} \\
n=16\end{array}$} & \multirow[t]{4}{*}{$\begin{array}{l}2.20(1.56) \mathrm{h} \\
n=16\end{array}$} & $9 \mu \mathrm{g} /$ day & $\begin{array}{l}174(124) \mathrm{pg} / \mathrm{mL} \\
n=9\end{array}$ \\
\hline & & & & $28 \mu \mathrm{g} /$ day & $\begin{array}{l}637(554) \mathrm{pg} / \mathrm{mL} \\
n=12\end{array}$ \\
\hline & & & & $5 \mu \mathrm{g} / \mathrm{m}^{2} /$ day & $\begin{array}{l}157(188) \mathrm{pg} / \mathrm{mL} \\
n=24\end{array}$ \\
\hline & & & & $15 \mu \mathrm{g} / \mathrm{m}^{2} /$ day & $\begin{array}{l}538(403) \mathrm{pg} / \mathrm{mL} \\
n=32\end{array}$ \\
\hline
\end{tabular}

$C_{s s}$ steady-state concentration after cycle 1 administration of $5 / 15 \mu \mathrm{g} / \mathrm{m}^{2} /$ day or $9 / 28 \mu \mathrm{g} /$ day of blinatumomab, $n$ sample size, $t_{1 / 2, Z}$ terminal half-life, $V_{z}$ volume of distribution based on terminal phase residuals (CWRES) and normalized prediction distribution errors (NPDE) showed random normal scatter around zero, with no specific pattern. The distribution of CWRES and NPDE versus time remained fairly constant, which demonstrated the absence of time-dependent pharmacokinetics. The mean of the NPDE for the serum concentrations was 0.037 (95\% CI 0.00862-0.0654) with a standard deviation (SD) of 0.891 (95\% CI 0.869-0.912). Although a slight bias of the pharmacokinetic variability was found, these results confirmed the appropriate model accuracy and precision because the $95 \%$ CI for the mean and SD of the NPDE for blinatumomab were very close to including 0 and 1 , respectively. There were no observations with absolute values of the CWRES $>6$, and hence there were no identified outliers.

The NONMEM ${ }^{\circledR}$-based final parameter estimates of the model are found in Table 3. Both fixed and random effects parameters were estimated with good precision [percentage relative standard error $(\% \mathrm{RSE}) \leq 16.1 \%$, and the condition number of 7 (calculated as the ratio of the highest to the lowest eigenvalue) indicated that the model was not ill conditioned.

\subsection{Internal Model Evaluation}

The pcVPC performed using the final model (Fig. 2) suggested that the developed model was appropriate in describing the time course of blinatumomab and its variability in a heterogeneous patient population across age groups. Since the pediatric pharmacokinetic parameters were supported by a relatively small number of patients who had sparse pharmacokinetic sampling, an additional pediatric-only pcVPC
Table 3 Parameter estimates and bootstrap analysis of the blinatumomab population pharmacokinetic parameters $(n=674)$

\begin{tabular}{lll}
\hline Parameter & $\begin{array}{l}\text { Final model (mean (RSE, \%) } \\
[95 \% \mathrm{CI}])\end{array}$ & $\begin{array}{l}\text { Non-parametric bootstrap: 500 } \\
\text { successful of 500 replicates } \\
\text { (median [95\% CI]) }\end{array}$ \\
\hline $\begin{array}{l}\text { Pharmacokinetic parameters } \\
\text { Volume (L) }\end{array}$ & $5.98(8.86)[5.14-6.98]$ & $5.99[5.14-6.98]$ \\
CL (L/h) & $2.22(2.95)[2.08-2.35]$ & $2.22[2.08-2.35]$ \\
$\quad$ Effect of BSA on CL $(\theta)^{\mathrm{a}}$ & $0.620(12.7)[0.46-0.76]$ & $0.625[0.463-0.756]$ \\
Inter-individual variability $(\% \mathrm{CV})$ & & \\
$\omega \mathrm{CL}$ & $47.6(16.1)[38.1-54.2]$ & $47.1[38.1-54.1]$ \\
$\omega \mathrm{EEPS}$ & $64.3(14.5)[55.0-73.0]$ & $64.4[55.0-73.0]$ \\
Residual variability $(\% \mathrm{CV})$ & $55.9(3.99)[52.6-61.5]$ & $56.1[46.3-75.6]$ \\
\hline
\end{tabular}

$B S A$ body surface area, $C I$ confidence interval obtained from NONMEM ${ }^{\circledR}$ asymptotic standard errors, $C L$ clearance, $C V$ coefficient of variation, $R S E$ relative standard error, $\omega C L$ inter-subject variability in CL, $\omega E P S$ inter-subject variability in residual variability

${ }^{\mathrm{a}} \mathrm{CL}_{\text {individual }}=\mathrm{CL} \cdot(\mathrm{BSA} / 1.876)^{\mathrm{Effect} \text { of BSA on } \mathrm{CL}}$ 


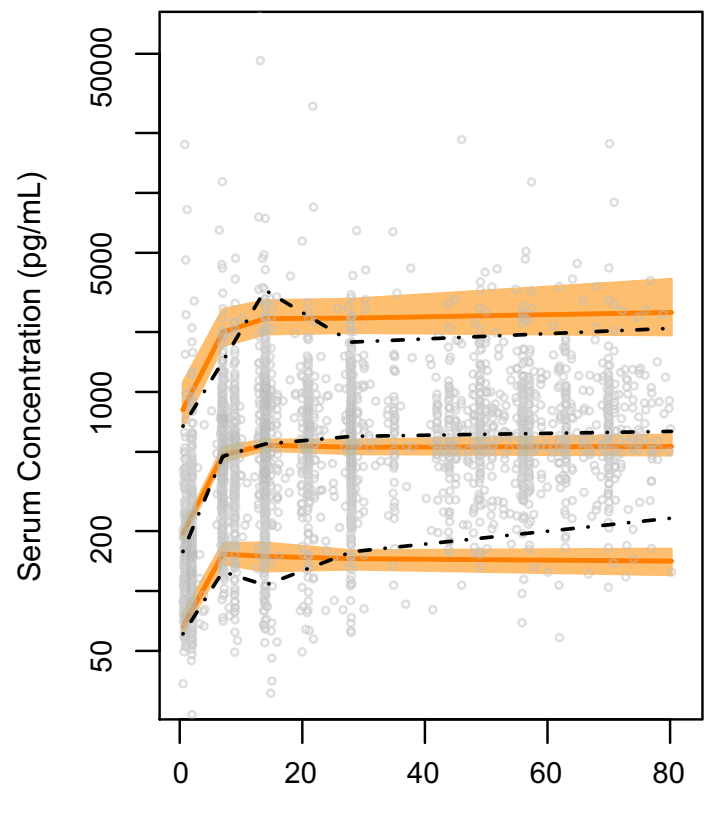

Time After First Dose (Days)

Fig. 2 Prediction-corrected visual predictive check, using 100 replicates, for all studies using the final model. Dashed black lines are the medians and $90 \%$ prediction intervals of observed data, which are shown as grey open circles. The shaded regions represent the $95 \%$ confidence intervals of the orange solid lines (median and $90 \%$ prediction intervals of simulated data)

showing adequate model performance in pediatrics is presented in Electronic Supplementary Material Fig. 6.
The non-parametric bootstrap distributions are summarized in Electronic Supplementary Material Fig. 7. The bootstrap tabulations of the model parameters are displayed in Table 3. Overall, the population estimates for the final model were very similar to the median of the bootstrap replicates, and were contained within the $95 \%$ CI obtained from the bootstrap analysis. The precision of the NONMEM ${ }^{\circledR}$ parameter estimates was good since the \%RSE for the fixed and random effects were lower than $17 \%$. This finding confirms the robustness of the NONMEM $^{\circledR}$-predicted parameter estimates.

\subsection{Simulations of Fixed, Body Surface Area-Based, and Per-Label Dosing Strategies}

During blinatumomab clinical development, BSA-based doses were converted to fixed doses in adult patients with a bodyweight of at least $45 \mathrm{~kg}$ to reduce dosing error rates, reduce drug waste, and improve dosing convenience. As illustrated in Fig. 3, the per-label dosing strategy resulted in similar exposures across the broad range of bodyweight. The median $C_{\mathrm{ss}}$ ranges across the $10 \mathrm{~kg}$ bins were as follows: (1) fixed dosing: 484-873 pg/mL; (2) BSAbased dosing: 391-567 pg/mL; and (3) per-label dosing: $391-603 \mathrm{pg} / \mathrm{mL}$. The interquartile ranges of blinatumomab $C_{\mathrm{ss}}$ for all patients $<45 \mathrm{~kg}$ for fixed, BSA-based, and perlabel dosing were 819,453 , and $453 \mathrm{pg} / \mathrm{mL}$, respectively, and for all patients $\geq 45 \mathrm{~kg}$ were 567,564 , and $567 \mathrm{pg} / \mathrm{mL}$,
Fig. 3 Simulated serum steady-state concentration for fixed dosing $(28 \mu \mathrm{g} /$ day, red dashed line), body surface area-based dosing $\left(15 \mu \mathrm{g} / \mathrm{m}^{2} /\right.$ day, blue dot-dashed line), and per label-based $\left(15 \mu \mathrm{g} / \mathrm{m}^{2} /\right.$ day for patients $<45 \mathrm{~kg}$, and $28 \mu \mathrm{g} /$ day for patients $\geq 45 \mathrm{~kg}$, green solid line) regimens in virtual patients having a broad range of bodyweights using a weight/ body surface area multivariate distribution. Body weights were binned for each $10 \mathrm{~kg}$ interval between 5 and $105 \mathrm{~kg}$, with 1000 patients simulated for each bin resulting in a total of 10,000 simulated patients. The top, middle, and bottom of the boxes are the third quartile, median, and first quartile, respectively. The whiskers are drawn to the nearest value not beyond $1.5 \times$ (upper hinge-lower hinge). $B S A$ body surface area

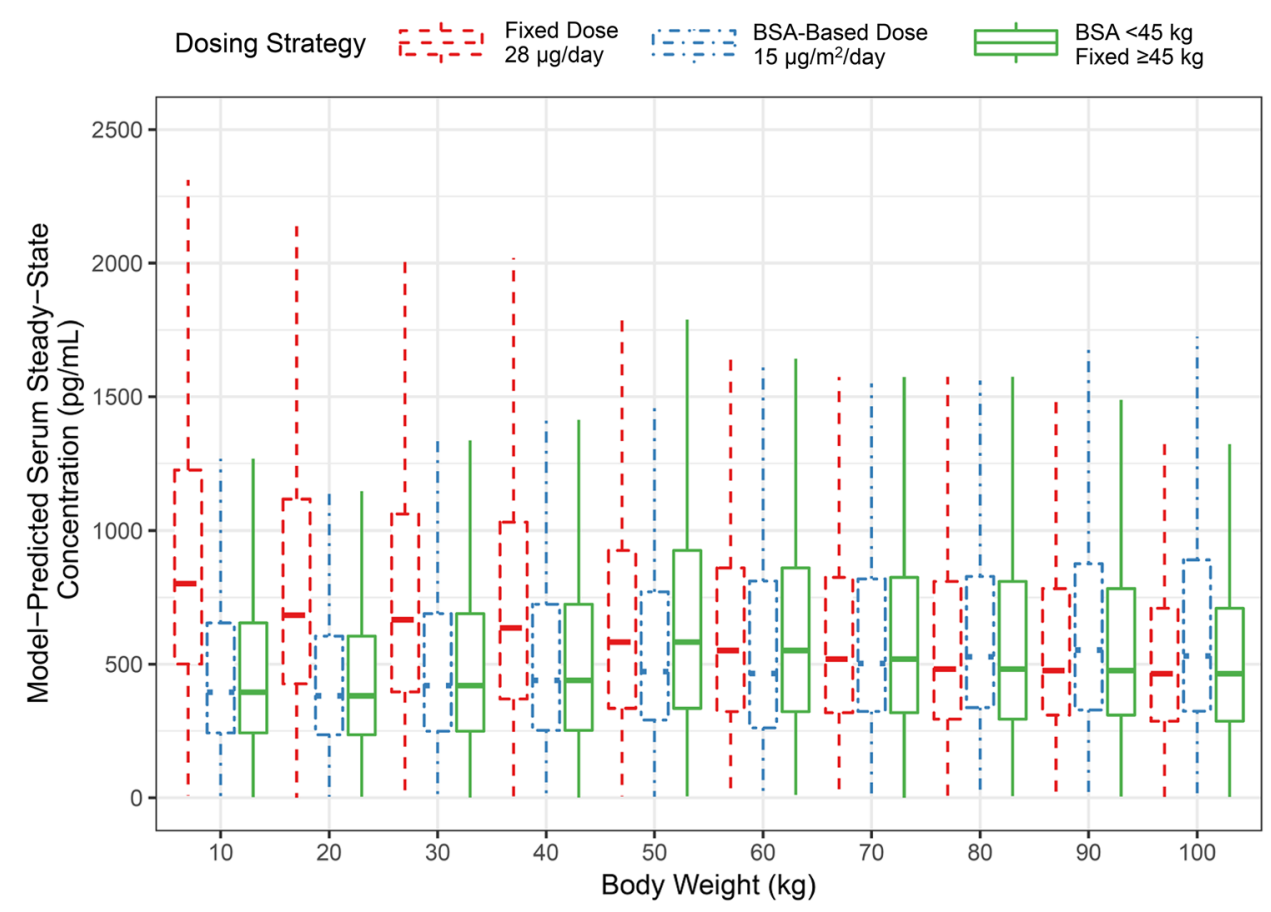


respectively. Note the broader range when using a fixed dosing approach for patients $<45 \mathrm{~kg}$.

\section{Discussion}

Blinatumomab showed linear kinetics across dose levels and over time based on population pharmacokinetic modeling. These results are consistent with the NCA and previous population pharmacokinetic modeling results [17]. In the population pharmacokinetic analysis, an open onecompartment linear pharmacokinetic model was suitable to describe the time course of blinatumomab concentrations, in different age groups, after cIV administrations of different doses in patients with hematologic malignancies, including R/R ALL, MRD-positive B cell precursor ALL, NHL, Ph+ ALL, and childhood R/R ALL.

While full-length immunoglobulin $\mathrm{G}$ antibodies with neonatal $\mathrm{Fc}$ receptor $(\mathrm{FcRn})$ domains typically have a terminal $t_{1 / 2}$ of approximately 23 days in humans [29], small fusion proteins such as BiTE $^{\circledR}$ antibody constructs, including blinatumomab, typically have shorter $t_{1 / 2}$ values, measurable in hours-comparable to many small molecules [30]. Blinatumomab, a BiTE ${ }^{\circledR}$ against CD19/ CD3, does not directly kill B cells, but rather it activates $\mathrm{T}$ cells and triggers an immune response which leads to $\mathrm{B}$ cell depletion-one activated $\mathrm{T}$ cell can kill more than one B cell-contributing to significant cytotoxicity at low $\mathrm{pg} / \mathrm{mL}$ concentrations [7]. Linear drug disposition, and not target-mediated drug disposition (TMDD), has been observed for blinatumomab within the range of doses studied, which contrasts with non-linear pharmacokinetics that may otherwise be associated with TMDD. Like monoclonal antibodies, BiTE $^{\circledR}$ antibody constructs have limited volumes of distribution, similar to plasma volume, and an elimination pathway consisting of rapid catabolism into simple amino acids [30]. According to the population pharmacokinetic model, the typical blinatumomab $V_{\mathrm{d}}$ value was estimated to be $5.98 \mathrm{~L}$, which is very close to plasma volume and similar to the values reported for other therapeutic fusion proteins [30]. The typical CL value was $2.22 \mathrm{~L} / \mathrm{h}$. Of note, the previous mixture model was no longer appropriate since the bimodal distribution filled into a single distribution with the additional data from the newly included studies.

The correlation coefficients for all covariates against CL were below 0.05 , suggesting that no clinically relevant covariates existed. However, the goodness-of-fit plots showed pediatric patients were not well-described by the model. With the inclusion of BSA on CL there was a significant improvement in the model fit for pediatric patients. Pediatric pharmacokinetics can be influenced by differences in blood flow, $\mathrm{pH}$, skin differences, absorption, body water and fat content, and circulating proteins, among other differences. This can impact (1) the volume of distribution; (2) intestinal, catabolic, liver, and kidney function; and (3) receptor-mediated endocytosis $[31,32]$. Pediatric dosage is often established empirically, based on age, bodyweight, BSA, or other differences such as fat composition. Further adjustments are often not necessary since the totality of CL and other differences are often simultaneously addressed [32]. The smallest BSA of $0.37 \mathrm{~m}^{2}$ in the pediatric population was associated with a $63 \%$ lower blinatumomab systemic CL relative to a patient with the median BSA $\left(1.88 \mathrm{~m}^{2}\right)$, supporting the use of BSA-based dosing in patients with lower body weight values. In contrast, the smallest and largest BSA values of 1.31 and $2.7 \mathrm{~m}^{2}$ in adults would be associated with a $20 \%$ reduction and a $25 \%$ increase, respectively, in blinatumomab systemic CL relative to a patient with the median BSA $\left(1.88 \mathrm{~m}^{2}\right)$. The magnitude of this effect is relatively low compared with the $48 \%$ unexplained between-subject variability in CL, and the $56 \%$ residual variability (which residual variability itself had a $64 \%$ between-subject variability) in blinatumomab pharmacokinetics. While dose adjustments based on BSA in patients $\geq 45 \mathrm{~kg}$ are not justifiable, it is important to note that the use of $15 \mu \mathrm{g} / \mathrm{m}^{2} /$ day BSA-based dosing in patients $<45 \mathrm{~kg}$ prevented excessive adverse events as determined in a phase I/II study of blinatumomab in pediatric patients [21], providing a strong incentive to retain this approach in lighter weight patients.

On average, for a patient with a BSA of $0.37,1.31$, 1.88 , or $2.7 \mathrm{~m}^{2}$, the blinatumomab $t_{1 / 2}$ was estimated to be $5.11,2.34,1.86$, or $1.49 \mathrm{~h}$, respectively. Therefore, the vast majority of the patients achieved $C_{\mathrm{ss}}$ within the first day of a 28-day cycle, regardless of BSA.

Other than BSA, none of the potential covariates (age, CrCL, sex, AST, ALT, total bilirubin, albumin, LDH, hemoglobin, dose level, and treatment cycle) were correlated with the between-patient variability of blinatumomab CL.

Clinical experience and further updates can be made when ongoing phase III studies in pediatric and adult patients are completed. Possible limitations to the analysis include lack of pharmacokinetic data in pediatric patients weighing $\geq 45 \mathrm{~kg}$ treated with the fixed-dose regimen, lack of pharmacokinetic data from adult patients weighing $<45 \mathrm{~kg}$ treated with a BSA-based dosing regimen, having fewer Black, Asian, and other non-White patients than White patients, and the lack of $\mathrm{T}$ cell and $\mathrm{B}$ cell baseline counts from all studies preventing their testing as a covariates in this analysis. Simulation results (Fig. 3) further indicated that the $C_{\mathrm{ss}}$ ranges were comparable across bodyweights when patients weighing $<45 \mathrm{~kg}$ were treated with the BSA-based dosing regimen or patients weighing $\geq 45 \mathrm{~kg}$ were treated with the fixed-dose regimen. The simulations also provide evidence that slight modifications to the weight 
cut-off would likely not change the drug exposure significantly. Since BSA-based dosing is not expected to offer benefit over fixed dosing, recommendation of the simpler fixed-dosing strategy for patients weighing at least $45 \mathrm{~kg}$ [8] is still reasonable.

\section{Conclusion}

An open one-compartment pharmacokinetic model with linear elimination was suitable to describe the time course of the serum blinatumomab concentration following cIV administration of doses ranging from 0.5 to $90 \mu \mathrm{g} / \mathrm{m}^{2} /$ day or two fixed-dose levels of 9 and $28 \mu \mathrm{g} / \mathrm{day}$ in patients with hematologic malignances, including patients with relapsing NHL, MRD-positive B-lineage ALL, R/R ALL (pediatric and adult), and R/R Ph+ ALL. The model has clinical applications in the dose and regimen selection in pediatric and adult patients over multiple indications. No covariates, other than BSA on CL, were identified as significant. While fixed blinatumomab dosing is acceptable in patients $\geq 45 \mathrm{~kg}$, BSAbased dosing should be considered for lower bodyweight patients to avoid excessive serum blinatumomab $C_{\mathrm{ss}}$ values.

Acknowledgments The authors thank James Ziobro (funded by Amgen Inc.) for medical writing assistance in the preparation of this manuscript. They would also like to thank the reviewers for their helpful comments and suggestions.

\section{Compliance with Ethical Standards}

Funding This study was funded by Amgen Inc. Amgen designed the study, and collected, analyzed, and interpreted the data.

Conflict of interest John D. Clements, Bianca Terminello, and Sameer Doshi are employees of and shareholders in Amgen Inc. Min Zhu and Mita Kuchimanchi were employees of and shareholders in Amgen Inc. when the analysis was conducted. Min Zhu is currently employed by Regeneron. Mita Kuchimanchi is currently employed by Biogen.

Ethical approval All procedures performed in studies involving human participants were in accordance with the ethical standards of the institutional and/or national research committee and with the 1964 Helsinki Declaration and its later amendments, or comparable ethical standards.

Informed consent Informed consent was obtained from all individual participants included in the studies.

Open Access This article is distributed under the terms of the Creative Commons Attribution-NonCommercial 4.0 International License (http://creativecommons.org/licenses/by-nc/4.0/), which permits any noncommercial use, distribution, and reproduction in any medium, provided you give appropriate credit to the original author(s) and the source, provide a link to the Creative Commons license, and indicate if changes were made.

\section{References}

1. Jabbour E, O’Brien S, Konopleva M, Kantarjian H. New insights into the pathophysiology and therapy of adult acute lymphoblastic leukemia. Cancer. 2015;121:2517-28.

2. Goto H. Childhood relapsed acute lymphoblastic leukemia: biology and recent treatment progress. Pediatr Int. 2015;57:1059-66.

3. Anderson KC, Bates MP, Slaughenhoupt BL, Pinkus GS, Schlossman SF, Nadler LM. Expression of human B cell-associated antigens on leukemias and lymphomas: a model of human B cell differentiation. Blood. 1984;63:1424-33.

4. Ginaldi L, De Martinis M, Matutes E, Farahat N, Morilla R, Catovsky D. Levels of expression of CD19 and CD20 in chronic B cell leukaemias. J Clin Pathol. 1998;51:364-9.

5. Harris NL, Stein H, Coupland SE, Hummel M, Favera RD, Pasqualucci L, et al. New approaches to lymphoma diagnosis. Hematol Am Soc Hematol Educ Progr. 2001;2001:194-220.

6. Horton HM, Bernett MJ, Pong E, Peipp M, Karki S, Chu SY, et al. Potent in vitro and in vivo activity of an $\mathrm{Fc}$-engineered anti-CD19 monoclonal antibody against lymphoma and leukemia. Cancer Res. 2008;68:8049-57.

7. Loffler A, Kufer P, Lutterbuse R, Zettl F, Daniel PT, Schwenkenbecher JM, et al. A recombinant bispecific single-chain antibody, CD19 $\times$ CD3, induces rapid and high lymphomadirected cytotoxicity by unstimulated T lymphocytes. Blood. 2000;95:2098-103.

8. Amgen Inc. Blinatumomab USPI. http://pi.amgen.com/unite d_states/blincyto/blincyto_pi_hcp_english.pdf. Accessed 6 Dec 2018.

9. Przepiorka D, Ko CW, Deisseroth A, Yancey CL, Candau-Chacon R, Chiu HJ, et al. FDA approval: blinatumomab. Clin Cancer Res. 2015;21:4035-9.

10. Zugmaier G, Gokbuget N, Klinger M, Viardot A, Stelljes M, Neumann S, et al. Long-term survival and T-cell kinetics in relapsed/ refractory ALL patients who achieved MRD response after blinatumomab treatment. Blood. 2015;126:2578-84.

11. Goebeler ME, Knop S, Viardot A, Kufer P, Topp MS, Einsele H, et al. Bispecific T-cell engager (BiTE) antibody construct blinatumomab for the treatment of patients with relapsed/refractory non-Hodgkin lymphoma: final results from a phase I study. J Clin Oncol. 2016;34:1104-11.

12. Gokbuget N, Dombret H, Bonifacio M, Reichle A, Graux C, Faul $\mathrm{C}$, et al. Blinatumomab for minimal residual disease in adults with B-cell precursor acute lymphoblastic leukemia. Blood. 2018;131:1522-31.

13. Klinger M, Brandl C, Zugmaier G, Hijazi Y, Bargou RC, Topp MS, et al. Immunopharmacologic response of patients with B-lineage acute lymphoblastic leukemia to continuous infusion of T cell-engaging CD19/CD3-bispecific BiTE antibody blinatumomab. Blood. 2012;119:6226-33.

14. Topp MS, Gokbuget N, Stein AS, Zugmaier G, O’Brien S, Bargou $\mathrm{RC}$, et al. Safety and activity of blinatumomab for adult patients with relapsed or refractory B-precursor acute lymphoblastic leukaemia: a multicentre, single-arm, phase 2 study. Lancet Oncol. 2015;16:57-66.

15. Topp MS, Gokbuget N, Zugmaier G, Klappers P, Stelljes M, Neumann S, et al. Phase II trial of the anti-CD19 bispecific T cellengager blinatumomab shows hematologic and molecular remissions in patients with relapsed or refractory B-precursor acute lymphoblastic leukemia. J Clin Oncol. 2014;32:4134-40.

16. Viardot A, Goebeler ME, Hess G, Neumann S, Pfreundschuh M, Adrian N, et al. Phase 2 study of the bispecific T-cell engager (BiTE) antibody blinatumomab in relapsed/refractory diffuse large B-cell lymphoma. Blood. 2016;127:1410-6. 
17. Zhu M, Wu B, Brandl C, Johnson J, Wolf A, Chow A, et al. Blinatumomab, a bispecific T-cell engager $\left(\right.$ BiTE $^{\circledR}$ ) for CD-19 targeted cancer immunotherapy: clinical pharmacology and its implications. Clin Pharmacokinet. 2016;55:1271-88.

18. Cockcroft DW, Gault MH. Prediction of creatinine clearance from serum creatinine. Nephron. 1976;16:31-41.

19. Kantarjian H, Stein A, Gokbuget N, Fielding AK, Schuh AC, Ribera JM, et al. Blinatumomab versus chemotherapy for advanced acute lymphoblastic leukemia. N Engl J Med. 2017;376:836-47.

20. Martinelli G, Boissel N, Chevallier P, Ottmann O, Gokbuget N, Topp MS, et al. Complete hematologic and molecular response in adult patients with relapsed/refractory Philadelphia chromosomepositive B-precursor acute lymphoblastic leukemia following treatment with blinatumomab: results from a phase II, single-arm, multicenter study. J Clin Oncol. 2017;35:1795-802.

21. von Stackelberg A, Locatelli F, Zugmaier G, Handgretinger R, Trippett TM, Rizzari C, et al. Phase I/phase II study of blinatumomab in pediatric patients with relapsed/refractory acute lymphoblastic leukemia. J Clin Oncol. 2016;34:4381-9.

22. Bargou R, Leo E, Zugmaier G, Klinger M, Goebeler M, Knop S, et al. Tumor regression in cancer patients by very low doses of a T cell-engaging antibody. Science. 2008;321:974-7.

23. Beal SL. Population pharmacokinetic data and parameter estimation based on their first two statistical moments. Drug Metab Rev. 1984;15:173-93.
24. Beal S, Sheiner LB, Boeckmann A, Bauer RJ. NONMEM user's guides. Ellicott City: Icon Development Solutions; 2009.

25. Bauer RJ. NONMEM users guide introduction to NONMEM 7.2.0. Ellicott City: Icon Development Solutions; 2011.

26. Post TM, Freijer JI, Ploeger BA, Danhof M. Extensions to the visual predictive check to facilitate model performance evaluation. J Pharmacokinet Pharmacodyn. 2008;35:185-202.

27. Efron B, Tibshirani R. An introduction to the bootstrap. London: Chapman and Hall; 1993.

28. Hunt CA, Givens GH, Guzy S. Bootstrapping for pharmacokinetic models: visualization of predictive and parameter uncertainty. Pharm Res. 1998;15:690-7.

29. Lobo ED, Hansen RJ, Balthasar JP. Antibody pharmacokinetics and pharmacodynamics. J Pharm Sci. 2004;93:2645-68.

30. Yuraszeck T, Kasichayanula S, Benjamin JE. Translation and clinical development of bispecific T-cell engaging antibodies for cancer treatment. Clin Pharmacol Ther. 2017;101:634-45.

31. Wagner J, Abdel-Rahman SM. Pediatric pharmacokinetics. Pediatr Rev. 2013;34:258-69.

32. Shi R, Derendorf H. Pediatric dosing and body size in biotherapeutics. Pharmaceutics. 2010;2:389-418. 